A N N A L E S Annales de Bretagne et des Pays de l'Ouest

\title{
L'Alliance démocratique et la recomposition des droites
}

Rosemonde Sanson

\section{(2) OpenEdition}

\section{Journals}

Édition électronique

URL : http://journals.openedition.org/abpo/1560

DOI : $10.4000 / a b p o .1560$

ISBN : 978-2-7535-1487-4

ISSN : 2108-6443

Éditeur

Presses universitaires de Rennes

Édition imprimée

Date de publication : 20 septembre 2002

Pagination : $95-108$

ISBN : 978-2-86847-768-2

ISSN : 0399-0826

\section{Référence électronique}

Rosemonde Sanson, «L'Alliance démocratique et la recomposition des droites », Annales de Bretagne et des Pays de l'Ouest [En ligne], 109-3 | 2002, mis en ligne le 20 septembre 2004, consulté le 19 avril 2019. URL : http://journals.openedition.org/abpo/1560 ; DOI : 10.4000/abpo.1560 


\title{
L'Alliance démocratique et la recomposition des droites
}

\author{
Rosemonde SANSON \\ Docteur d'État \\ Maître de conférences à l'Université de Paris 1 \\ Centre de recherches d'histoire du XIX ${ }^{\mathrm{e}}$ siècle
}

Avec un peu de provocation, il est licite d'avancer que l'Alliance démocratique - fondée en 1901 par Adolphe Carnot, au temps du gouvernement de défense républicaine dirigé par Waldeck-Rousseau, et présidée, à partir de 1933, par Pierre-Étienne Flandin - a plus constitué un obstacle à la recomposition des droites, entre 1933 et 1940, qu'elle n'a contribué à son élaboration. Le nouveau président, après une période de déliquescence du mouvement, ambitionne de le restructurer en un "grand parti du centre ". À aucun moment, ni Pierre-Étienne Flandin ${ }^{1}$, ni ses amis, inspirateurs de la ligue officielle du parti, n'ont prétendu présenter l'Alliance démocratique comme une force intégrante des droites. Il n'empêche. A contrario, le président du parti se donne à voir, au temps du Front populaire, comme le leader de l'opposition aux gouvernements Blum. Puis, face aux exigences d'Hitler, lors de la conférence de Munich à l'automne 1938, il apparaît comme le tenant le plus extrémiste du clan des pacifistes de droite. De plus, pendant toute la période, le président de l'Alliance et les alliancistes axent leur propagande autour de l'antibolchevisme, thème fédérateur pour les droites.

Certes la ligne officielle du parti est, à plusieurs reprises, fortement contestée par certains de ses membres, partisans d'un système bipolaire, droite contre gauche. Il faudra y revenir. Il conviendra aussi de rechercher les motivations profondes de la politique flandiniste, marquée à l'aune de la bivalence, voire de l'ambivalence.

La question de l'Alliance démocratique et de la recomposition des droites sera donc abordée autour d'une problématique double : l'Alliance agent de la décomposition des droites, et l'Alliance actrice de la mobilisation contre les gauches.

1. SANSON, Rosemonde, L'Alliance républicaine démocratique, une formation de centre, thèse d'État, 2000, Paris 4, à paraître fin 2002 aux PUR. " ARD ou AD, Alliance démocratique ", SIRINELLI, Jean-François (dir.), Dictionnaire de la vie politique française au $X X^{e}$ siècle, PUF, 1995. 


\section{Le rejet de la mobilisation à droite}

« La France est un pays de juste-milieu. L'Alliance représente précisément ce juste-milieu. Elle essaiera de sauver, dans la crise qui se prépare, le régime républicain ", précise Pierre-Étienne Flandin lors du congrès refondateur de l'Alliance le 23 mars 1933. Plus avant dans son discours, il dit muer l'association en parti afin " de donner aux partis du centre la force qu'il faut bien constater qu'ils ont momentanément perdue ».

L'emploi du pluriel traduit sa volonté de structurer la nébuleuse des républicains de gauche, aux sensibilités politiques voisines. D'ailleurs, l'Alliance devient « le parti autonome des républicains de gauche et des républicains radicaux ${ }^{2}$ ». On remarquera qu'il emploie les vocables centre, républicains de gauche. Ce faisant, il donne de l'Alliance démocratique et des alliancistes une représentation plus à gauche que la réalité politique. En tout cas, il n'entend pas la rattacher à la famille des droites. Certes, ce gauchissement est typique de la période. Il importe, néanmoins, de considérer l'image que les politiques veulent donner d'eux-mêmes.

\section{Pour une concentration républicaine}

Au nom des principes évoqués, Pierre-Étienne Flandin s'engage, indirectement ou personnellement, en faveur d'une politique de concentration républicaine. Les instances dirigeantes du parti rendent compte de cette ligne officielle dans des éditoriaux et des articles publiés dans l'Alliance démocratique ou $A D$, l'hebdomadaire du mouvement. Ils passent plus volontiers sous silence les contestations soulevées par une telle politique. La presse d'opposition et des indicateurs de la police pallient les lacunes.

À la suite du 6 février 1934, le gouvernement Doumergue qui comprend trois membres éminents de l'Alliance (Barthou, Flandin, Rollin) est perçu comme un temps de "trêve des partis ". Le président salue avec satisfaction " les liens cordiaux " de nouveau tissés entre radicaux et alliancistes. Il affirme :

" J'ai, en effet, constamment soutenu qu'il était nécessaire que se dégage dans ce pays une majorité gouvernementale stable, appuyée sur ses deux piliers que sont, d'une part, le parti radical et radical-socialiste, et, d'autre part, l'Alliance démocratique ${ }^{3}$."

Il y voit la marque d'une fidélité au passé, mais surtout un gage pour l'avenir.

2. FLANDIN, Pierre-Étienne, Paix et Liberté (L'Alliance démocratique à l'action), Éditions de l'AD, 1938. BN : Fonds Alliance démocratique, discours prononcé le 29 mars 1933 au congrès national extraordinaire de l'Alliance.

3. Arch. dép. de Gironde : le Congrès d'Arras, publication de l'AD, discours prononcé au congrès (2, 3 et 4 novembre 1934). 
Lorsque Pierre-Étienne Flandin est appelé à succéder à Doumergue au début de novembre 1934, le collectif de l'Alliance, dans l' $A D$, approuve son président d'avoir fortifié la " trêve des partis ". Édouard Herriot et Louis Marin ne reçoivent-ils pas des portefeuilles de ministre d'État? Quant à Joseph-Claude Gignoux, allianciste reconnu, il écrit dans la Journée industrielle : " Ce sera une belle expérience de voir le médecin désigné de notre économie nationale, ayant exactement diagnostiqué le mal, organiser maintenant le traitement ${ }^{4}$."

En revanche, l'entrée de Pierre-Étienne Flandin, de René Besse et d'André Beaugritte, dans le cabinet du radical Albert Sarraut, constitué de 24 janvier 1936, soulève une houle au sein du parti. Quelques jours plus tôt, le rassemblement populaire ne vient-il pas, en effet, de publier son programme? Flandin doit se justifier. Selon lui, l'acceptation des Affaires étrangères n'aliène en rien sa liberté d'action de président du parti pour combattre le Front populaire, aux élections prochaines. Le comité directeur donne son aval à Flandrin et aux deux autres députés participant au gouvernement. L'ordre du jour inscrit cette collaboration dans le droit fil d'une politique de centre ${ }^{5}$. Tel n'est pas le sentiment des alliancistes hostiles à la participation au gouvernement Sarraut. Les opposants sont tenus par des préoccupations électoralistes et doctrinales. Leur prise de position témoigne aussi de leur ancrage à droite.

Afin de discuter d'une impossible compromission avec les radicauxsocialistes fourvoyés, début 1936, dans le Front populaire aux côtés des socialistes et des communistes, Marcel Héraut et Charles Reibel, respectivement députés de la Seine et de la Seine-et-Oise, demandent la réunion d'un congrès extraordinaire. De fait, ils espèrent aboutir à une condamnation de la décision du président de l'Alliance. Ils menacent même de faire scission. Plus modéré, le vice-président André de Fels, proche pourtant de Flandin, suggère à celui-ci de résilier, un temps, la présidence du mouvement. Ainsi, les candidats de l'Alliance auraient les coudées plus franches pour combattre ceux du parti valoisien affiliés au Front populaire ${ }^{6}$. Finalement la crise a tourné court, non sans laisser cependant des cicatrices.

La victoire électorale des candidats du Front populaire, la formation du gouvernement Blum rendent non avenue la politique concentrationniste. L'espoir renaît dès l'échec de Léon Blum, en juin 1937, et son remplacement par le radical-socialiste Camille Chautemps. Alors, la direction de l'Alliance pratique, si l'on ose écrire, la tactique de la main tendue. Tout en déplorant le climat d'insécurité et d'incertitude que font régner les partis extrémistes, le collectif rédactionnel avance dans l'AD:

4. Rapporté dans $A D$, " Le ministère Flandin ", 14 novembre 1934.

5. Cf. plusieurs numéros de l'AD, janvier-février 1935 .

6. Arch. Préfecture de Police, B/A 1897. Un carton concernant l'Alliance démocratique entre les deux guerres : renseignements des indicateurs de police, janvier et mars 1936; en particulier, notes du 27 janvier 1936 et du 4 mars 1936. 
" Notre parti, qui a toujours combattu la politique des deux blocs et préconisé la réconciliation des républicains, serait mal venu de se dérober le jour où il serait appelé à participer à un large rassemblement qui, de surcroît, apparaîtrait sans doute au pays comme la suprême chance des salut ${ }^{7}$."

Certes, lorsque Léon Blum échoue une deuxième fois et laisse la place à Daladier, les alliancistes - à l'exception de Paul Reynaud, marginal par rapport à la ligne directrice du parti - ne sont pas appelés à l'effort de redressement. Pourtant, les flandinistes apportent un soutien conditionnel, puis franc, à Édouard Daladier. Un nouveau rapprochement semble s'esquisser entre " républicains du milieu ${ }^{8}$ ", les alliancistes, et radicaux-socialistes. Il est vrai que ces derniers opèrent alors un sérieux recentrage.

\section{Contre les projets de recomposition à droite}

Corrélativement, le comité directeur de l'Alliance démocratique, conséquent avec la ligne concentrationniste, repousse, par trois occasions au temps du Front populaire, les offres d'entente avec des formations situées plus à droite ou considérées comme telles.

La première tentative concerne la préparation des législatives du printemps 1936. Elle émane d'Henri de Kérillis et des républicains nationaux, avec d'ailleurs Paul Reynaud et André Maginot. Le directeur de L'Écho de Paris puis de L'Époque profite, sans nul doute, des troubles provoqués au sein du parti par l'entrée de Flandin dans le cabinet Sarraut pour inviter les alliancistes à participer au comité d'arbitrage qu'il met en place en région parisienne en vue des prochaines élections législatives. Il prônait une discipline de désistement entre républicains nationaux et républicains modérés, au sens large du terme, pour faire barrage aux candidats de Front populaire. Par la plume de René Besse, un allianciste partisan de la concentration républicaine, les instances dirigeantes de l'Alliance opposent une fin de non-recevoir ${ }^{9}$. Après tergiversations, on aboutit à une situation ambiguë : des alliancistes participent au comité Kérillis; mais, officiellement, l'Alliance se présente avec son programme et entend conduire sa propre campagne électorale.

Ainsi les rédacteurs de l'appel aux électeurs publié dans l'AD du 10 avril 1936 précisent-ils à l'adresse des sympathisants :

"Condamnant toute politique qui diviserait la France en deux blocs destinés à se heurter violemment et qui conduiraient à la guerre civile, vous vous prononcerez pour une politique d'union des républicains et de réconciliation nationale $[\ldots]$. Aux coalitions d'intérêt purement électoral, condam-

7. "Le ministère Chautemps ", $A D, 16$ juillet 1937.

8. SANSON, Rosemonde, "L'Alliance démocratique ", REMOND, René, BouRdin, Janine (dir.), La France et les Français au temps du gouvernement Daladier, Presses de Sciences Po, 1978.

9. "René Besse précise la position de son parti ", $A D, 28$ février 1936; cf. aussi APP B/A 1897, et communication de Jean Vavasseur-Desperriers. 
nées à se dissoudre demain dans l'impuissance d'agir, vous opposerez la concentration de toutes les forces républicaines ${ }^{10}$."

Contre toute évidence, les responsables de l'Alliance croient prudent de ménager sinon les radicaux-socialistes du moins leurs électeurs potentiels, et misent sur la dislocation rapide du rassemblement populaire.

Au lendemain des élections, Pierre-Étienne Flandin et ses affidés caressent le projet de constituer un grand groupe de centre gauche à la Chambre comprenant les élus du parti, mais aussi des radicaux-socialistes dissidents ainsi que les membres de la Fédération républicaine. Il prendrait place aux côtés d'un groupe d'extrême gauche, d'un groupe de gauche et d'un groupe de centre droit. Le projet avorte. De plus, tous les députés alliancistes ne se sont pas ralliés au groupe créé par le parti. Constatant cela, Louis Marin, de la Fédération républicaine, lance l'idée d'une " délégation permanente des groupes de la minorité ", sorte d'intergroupe parlementaire. Léon Baréty, président du nouveau groupe des Républicains de gauche et radicaux, incarnation de l'Alliance à la Chambre, répond positivement. Mais seuls quelques députés de l'aile droite, tel Creyssel, ont vraiment participé aux rencontres. Un peu excédé, Louis Marin réitère l'invite, en particulier pendant les vacances parlementaires de 1937. Chautemps étant alors au pouvoir, Léon Baréty préfère ne pas répondre par lettre. Pressé par PierreÉtienne Flandin auprès de qui Louis Marin s'est plaint de la discourtoisie des alliancistes, Léon Baréty finit par envoyer une missive. La réponse est, cette fois, négative :

" Notre groupe, écrit-il, n'avait point en principe l'intention de participer à ces réunions permanentes de certains groupes de la minorité, si les circonstances ne l'exigeaient pas ${ }^{11}$."

Dès mars 1937, Jacques Doriot, député de Saint-Denis et leader du Parti populaire français ou PPF, échafaude un "front de la liberté " contre " la dictature soviétique ". Il envisageait de regrouper les formations partisanes issues des ligues, et aussi les mouvements telles l'Alliance démocratique et la Fédération républicaine. L'appel est renouvelé solennellement, en mai, au cours d'un grand meeting du PPF.

Le comité directeur de l'Alliance refuse toute participation au Front de la liberté. Il argue de deux motifs essentiels. D’abord, les alliancistes se présentent comme les "défenseurs de toutes les libertés ". Pourquoi, dès lors, adhérer à un Front de la liberté? Ensuite, il dit craindre qu'un tel regroupement rende irrémédiables les divisions entre forces politiques françaises. Le secrétaire général du mouvement, André Terrasse, justifie : " Il eût été mauvais que les partis d'opposition formassent, en face du Front popu-

10. " Appel aux électeurs ", $A D, 10$ avril 1936.

11. VAVASSEUR-DESPERRIERS, Jean, Culture, structures, stratégie d'une organisation de la Droite parlementaire entre les deux guerres, la Fédération républicaine de 1919 à 1940, thèse d'État, Lille, 1999. Voir aussi BN, Fonds Alliance démocratique, lettres de Louis Marin, de Pierre-Étienne Flandin et Léon Baréty, 22 mai, 12 juillet et 15 juillet 1937. 
laire en voie de désagrégation, un bloc adverse ${ }^{12}$. " Un autre argument est passé sous silence, mais a dû jouer : que Doriot soit un ancien membre du parti communiste. Une fois encore, il y eut des dissidents. Ainsi, Jean-Louis Tixier-Vignancour, situé à l'aile droite de l'Alliance, adhère au Front de la liberté, mais il le fait à titre personnel, n'engageant pas le parti. Les instances dirigeantes de l'Alliance n'ont donc pas renoncé à ce qu'elles croient être la vocation de leur parti, être l'axe d'une politique de concentration républicaine, ce qui implique de ne pas se compromettre avec les droites fascisantes, ou même de se confondre avec les républicains de droite.

En février 1939, à l'inverse, les congressistes de la fédération de Seine-etOise de l'Alliance, réunis à Versailles, émettent le vœu de former « un grand front républicain " regroupant les élus de la Fédération républicaine jusqu'à ceux de l'Union socialiste; l'Alliance en serait l'axe ${ }^{13}$. D'autres, moins « regardants ", résument leur perspective en cette formule : d'Herriot à Henriot.

La réitération d'une même ligne politique à une époque d'incontestable bipolarisation est-elle une preuve d'aveuglement, d'anachronisme ${ }^{14}$ ? Un peu, sans doute, mais il est d'autres facteurs explicatifs.

\section{Les motivations profondes}

Le positionnement politique de Pierre-Étienne Flandin et de la majorité des alliancistes découle de quatre principaux enchaînements causaux.

Tout d'abord, nombre d'alliancistes gardent et entretiennent la nostalgie des origines. Le président du parti aime à situer son parti dans la continuité du modèle waldeckiste. Il s'identifie lui-même à un Waldeck-Rousseau unissant en son gouvernement de défense républicaine des républicains modérés, des radicaux-socialistes et même deux socialistes non collectivistes. Est-ce fondé dans le contexte des années trente? Sur ce point, on pourrait, peut-être, taxer les flandinistes d'anachronisme.

Seulement, il convient de tenir compte d'une autre fidélité, à un passé récent cette fois. C'est la seconde explication de leur comportement. $\mathrm{Au}$ congrès tenu à Dijon en 1929, deux théories se sont affrontées : celle de Paul Reynaud, défenseur de la formation d'un grand parti conservateur à l'anglaise, et de la politique bloc contre bloc; celle de Pierre-Étienne Flandin, partisan d'une Alliance, formation politique de concentration républicaine. On sait que l'année suivante, au congrès d'Angers en 1930, les alliancistes se sont prononcés pour une telle ligne politique et la constitution d'un grand parti centriste, rejetant, par là même, la théorie des deux blocs ${ }^{15}$. Les

12. " Réponse du comité directeur à Jacques Doriot ", $A D, 4$ juin 1937; TERRASSE, André, "Les événements marchent ", 11 juin 1937 et APP B/A 1897, note du 4 juin 1937.

13. " La Fédération de Seine-et-Oise ", $A D, 17-24$ février 1939.

14. Cf. AUDIGIER, François : « l'Alliance démocratique de 1933 à 1937 ou l'anachronisme en politique ", Vingtième siècle, juillet-septembre 1995.

15. Congrès national de Dijon et Congrès national d'Angers, deux brochures de l'AD, l'une conservée à la BN, Fonds Flandin, carton 91, l'autre à la bibliothèque du Sénat. 
années suivantes, Flandin, ayant pris la direction de l'Alliance démocratique, demeure fidèle à lui-même et les instances dirigeantes ne se déjugent pas. Peut-on leur en faire grief? En plein exercice du Front populaire et bien que le combattant, la direction de l'Alliance refuse encore de choisir :

" Le premier devoir de tout citoyen, lit-on dans l'AD, est de lutter contre le communisme, mais faut-il, sous prétexte d'échapper à l'emprise bolchevique, sombrer dans une autre tyrannie qui s'appelle la dictature? L'ordre, la prospérité et la paix peuvent s'obtenir dans la liberté ${ }^{16}$."

À la formule " ni réaction, ni révolution " s'est substituée celle de " ni bolchevisme, ni fascisme ". Peut-on y discerner un manque de pragmatisme, d'adaptabilité au contexte historique? Au contraire. La prise de position s'explique par deux considérations à moyen terme : il faut se débarrasser du Front populaire puis, sur les ruines opérées par le gouvernement de Blum " appuyé sur le communisme ", reconstruire la république parlementaire. Pour y parvenir, le soutien des radicaux-socialistes, pour l'heure fourvoyés dans une voie contraire à leur concept, s'avère obligatoire. Il convient donc de les ménager.

De fait, les dirigeants de l'Alliance, troisième facteur d'explication, se présentent comme les continuateurs des "républicains de gouvernement ". Ils estiment que les alliancistes ont vocation à exercer le pouvoir ou, pour le moins, à participer à des gouvernements d'union nationale ou de concentration républicaine. Ils n'ont pas renoncé à jouer un rôle modérateur dans la vie politique et à influer sur son cours. La France n'est-elle pas, le plus souvent, gouvernée par des coalitions de partis centristes, et non par les seules forces de droite ou les seules familles de gauche? La bipolarisation, par ailleurs, leur paraît source d'instabilité gouvernementale car non représentative des électeurs, en majorité hostiles aux extrêmes dans une France au multipartisme ${ }^{17}$.

Enfin, les flandinistes ont conscience de la place primordiale, incontournable, du parti radical et radical-socialiste dans la vie politique française. Ce parti est d'ailleurs traversé de courants, dont l'un penche à gauche et l'autre vers le centre ${ }^{18}$. Dans un discours prononcé à Montauban à l'automne 1937, Albert Sarraut, ministre d'État dans le cabinet Chautemps, fait écho aux propos d'un Pierre-Étienne Flandin. Il déclare en effet :

"L'existence d'un parti radical évite à la France la division redoutable en deux blocs de droite et de gauche [...]. Il est l'amortisseur [...]. Par ce rôle et cette position régulatrice, il est, au premier chef, une garantie contre la guerre civile. [...]. » Plus avant dans son discours, il va même, lui aussi, étiqueter son parti, de " parti du juste-milieu ${ }^{19}$."

Deux centrismes donc, faits pour collaborer.

16. "Contre les deux mystiques ", $A D, 22$ janvier 1937.

17. Cf. « Débats du Congrès de Bourg-en-Bresse ", $A D, 13$ novembre 1936.

18. BERnsteIn, Serge, Histoire du parti radical, t. II, Presses de la Fondation des Sciences politiques, 1980.

19. Rapporté dans BERnSTEIN, Serge, op. cit. 
Au vrai, malgré nombre de divergences, radicaux-socialistes et alliancistes possèdent un fond idéologique commun : l'attachement, certes à des degrés divers, au républicanisme parlementaire, à la laïcité, au libéralisme. De plus, les deux partis entendent représenter et défendre les classes moyennes ${ }^{20}$.

Serge Berstein a pertinemment démontré ce dernier point dans sa thèse sur le parti radical pendant l'entre-deux-guerres. On ne l'avait pas encore suffisamment fait remarquer en ce qui concerne l'Alliance. Au congrès refondateur du mouvement en 1933, Pierre-Étienne Flandin avance que l'Alliance démocratique est le parti du « Tiers état ». Il précise alors :

" Nous sommes le parti de ces petits bourgeois, surtout de ces petits bourgeois de province. [...]. Nous sommes le parti des ouvriers intelligents qui veulent s'élever par leur travail et leurs vertus, [...] dédaigneux d'une mystique révolutionnaire. [...]. C'est aussi le parti de ces paysans laborieux et tenaces $[\ldots]^{21}$."

En écho, les alliancistes, réunis en congrès à Bourg-en-Bresse en novembre 1936, critiquent la politique financière et économique du Front populaire, car elle lèse les classes moyennes. Or ils estiment nécessaire de prendre " la défense des classes moyennes, ossature de la nation sur lesquelles retombe, sans compensation, le poids des réformes réalisées et qui doivent être en mesure de rétablir l'équilibre rompu à leur détriment ${ }^{22}$ ". Rhétorique facile? Peut-être. En tout cas, alliancistes et radicaux-socialistes s'accordent pour reconnaître en ces classes moyennes l'assise de la société et le fondement de la démocratie en France.

\section{L'Alliance, pivot d'une recomposition des droites?}

À l'inverse de ce qui vient d'être démontré, le président de l'Alliance et ses amis, ainsi qu'une minorité d'alliancistes, chacun à sa manière, ont mené une lutte très ferme contre les gauches. Bref, les alliancistes, de façon involontaire ou voulue, ont contribué à la recomposition des droites.

\section{L'existence d'un courant droitier}

L'Alliance démocratique n'est pas un parti monolithe. Un clivage sépare une aile gauche, disons flandiniste, et une aile droite, gagnée à la contestation contre la politique dessinée par le président du mouvement.

Discernable dès les congrès de Dijon et d'Angers, cette division idéologique - qu'ont voilée d'artificiels votes à l'unanimité, selon la tradition du parti - s'accroît dans le climat passionné du Front populaire.

20. SANSON, Rosemonde, "Les relations entre l'Alliance démocratique et le parti radical pendant l'entre-deux-guerres ou l'existence d'un centre ", MoLLER, Horst, KITTEL, Demokratie in Deutschland und Frankreich 1918-1933/1940, Munich, 2002, p. 203-218.

21. Flandin, Pierre-Étienne, Paix et Liberté, un programme d'action pour l'Alliance, Éditions de l' $A D, 1933$.

22. $A D, 13$ novembre 1936. 
Au lendemain des élections de 1936, Georges Portmann de la Fédération girondine, nettement radicalisante, qui a regagné le giron de l'Alliance l'année précédente au congrès de Bordeaux en 1935, confesse son inquiétude à son ami Flandin. L'avortement d'un grand groupe centriste ne signe-t-il pas le positionnement des députés trop à droite?

"L'Alliance démocratique, lui écrit-il, me semble rejetée fort à droite, et c'est là une situation qui ne doit pas cadrer tout à fait avec vos désirs, ni les miens ${ }^{23}$."

A contrario, la fraction droitière du mouvement a plaidé, lors de la campagne électorale, pour un rapprochement avec les candidats de la Fédération républicaine de Louis Marin, et pour une entente de désistement avec les "républicains nationaux de droite ". La seule planche de salut, selon elle, consiste à un regroupement de tous les républicains nationaux, anticommunistes ${ }^{24}$. Idéologiquement, elle se sent proche d'eux.

Aux congrès du parti tenu à Bourg-en-Bresse en novembre 1936, puis à Nice l'année suivante, une minorité s'exprime, contestant toute concession faite ou au bolchevisme et à ses affidés, ou au parti radical-socialiste qui a opté pour le camp révolutionnaire. À Bourg-en-Bresse, la signature du parti franco-soviétique et l'adage " ni bolchevisme ni fascisme " sont violemment contestés. Certains intervenants se prononcent contre tout compromis avec les forces du mal et se posent en champion de l'anticommunisme. La signature du pacte des forces de gauche leur paraît ouvrir la voie à la révolution bolchevique, et ils stigmatisent la politique de balancier habituelle à l'Alliance. Le temps n'est plus du fleuret moucheté. La critique se fait acerbe. Évidemment les journaux témoignent des débats houleux. Manifestement, certains adhérents du mouvement présidé par Flandin s'opposent à leur leader et se situent dans la famille des droites.

À Nice, en novembre 1937, Léon Baréty croit bon, en clôture du congrès annuel, de défendre la ligne officielle du parti. "En regard de tous les fascismes, quel qu'en soit le contenu, l'Alliance, affirme-t-il, entend constituer le bloc de la liberté et de la raison. " C'est une réponse implicite aux contestataires du parti qui ont estimé dangereuse la prise de position conciliante à l'égard du gouvernement Chautemps.

"Qu'arrivera-t-il, interroge Georges Prade, président du comité du quatorzième arrondissement de Paris, si, dans quelques semaines, quelques mois, le gouvernement Chautemps est obligé de se retirer par suite de l'opposition entre les partisans de l'orthodoxie financière et les tenants de la prodigalité? [...] Je pense qu'il serait redoutable de prêter notre doctrine et nos hommes à une aventure sans lendemain ${ }^{25}$."

Des alliancistes - tels Charles Trochu, Marcel Héraut ou encore JeanLouis Tixier-Vignancour - n'hésitent pas à s'engager aux côtés des initia-

23. BN, Fonds Alliance démocratique, Lettre de Portmann à Flandin, 28 mai 1936.

24. APP B/A, carton 1897, notes de janvier et février 1936.

25. "Le comité directeur », $A D, 26$ novembre 1936. 
teurs de regroupement de droite. D'une façon plus générale, les jeunes ne répugnent nullement à convoquer à leurs réunions des orateurs adeptes des mouvements fascisants, voire de l'Action française. Plus sociaux, ces jeunes manifestent cependant une position politique plus droitière ${ }^{26}$.

Incontestablement, des alliancistes se posent en tenants d'une droite unitaire contre le Front populaire et les familles politiques qui le composent, radicaux-socialistes compris. Pourtant, Pierre-Étienne Flandin et ses partisans apparaissent comme de fermes opposants au Front populaire, au danger bolchevique au point de modifier leurs positions en politique extérieure.

\section{Flandin, leader de l'opposition au Front populaire}

Dès la formation du cabinet conduit par Léon Blum, l'Alliance, par le truchement de son comité directeur, s'érige en rempart contre ce gouvernement, sous l'emprise, selon elle, de Moscou. À la Chambre, Pierre-Étienne Flandin intervient plusieurs fois pour attirer l'attention sur le danger des mesures arrêtées par les socialistes. Le président de l'Alliance fait figure de leader de l'opposition au Front populaire, en tout cas sous les deux gouvernements conduits par Léon Blum.

Dès le $1^{\text {er }}$ juillet 1936, le comité directeur - ce jour-là sous la présidence de Léon Baréty - invite les élus et les adhérents de l'Alliance démocratique,

" à redoubler d'efforts pour opposer à la propagande révolutionnaire du parti communiste le rassemblement des forces françaises, décidées à lutter contre le chômage et l'appauvrissement de la nation, pour la liberté individuelle, la protection de la famille, le respect de la propriété, l'ordre dans la rue, le progrès social, le culte de la patrie et la paix ${ }^{27}$ ".

Le programme mêle affirmation des valeurs et des idéaux véhiculés par la culture des droites.

Pierre-Étienne Flandin multiplie les discours pour stigmatiser l'action du Front populaire, qui, selon lui, conduit à l'étatisation et à la socialisation progressive des moyens de production et de distribution et aggrave le déficit budgétaire. Cet adversaire de la dévaluation ne cesse de mettre l'accent sur les méfaits d'une telle décision. Le comité directeur, à plusieurs reprises vote, en conséquence, des ordres du jour désapprobateurs et met en garde

" tous les Français [...] contre une politique d'illusion, de lutte de classes et de vacance de leur légalité aussi dangereuse pour la paix extérieure que pour la paix intérieure ${ }^{28}$."

26. APP B/A, carton 1897 et aussi SANSON, Rosemonde, "Les jeunesses de l'Alliance démocratique ", Recherches contemporaines, 2001-2002, université de Paris 10-Nanterre.

27. " Le comité directeur ", $A D, 4$ juillet 1936.

28. "Réunion du comité directeur ", $A D, 18$ décembre 1936. 
Les interventions du président ou les mises au point des instances dirigeantes s'assortissent, attirant l'attention sur les graves responsabilités de soutenir Blum.

Au début de l'année 1937, l'Alliance démocratique, publie quatre brochures, au prix modique d'un franc chacune. Ce sont autant de brûlots contre la politique économique du Front populaire. Elles ont pour titres : Le Front populaire contre le franc, Le Front populaire contre l'agriculture, Le Front populaire contre le commerce et l'industrie. La quatrième transcrit le texte de l'interpellation que Pierre-Étienne Flandin a fait à la Chambre des députés, le 26 février 1937. Son intitulé suggère le contenu : Le Front populaire nous conduit à la catastrophe ${ }^{29}$. En réponse à la " pause " annoncée par Léon Blum, l'orateur dresse le bilan catastrophique d'un an de Front populaire, tant sur le plan financier et budgétaire, que social et national. Il rétorque au chef du gouvernement :

"Vous vous flattez non seulement d'éviter la catastrophe, mais de mener la démocratie vers de magnifiques destins. Nous avons, nous, au contraire, la conviction que vous menez le pays à la catastrophe et que le régime républicain périra si vous persévérez dans la voie où vous vous êtes engagé. "

Il se présente aussi en défenseur des intérêts du Français moyen. Ce faisant, il préconise la collaboration de classes :

" Il ne peut être question de brimer la classe ouvrière. Mais c'est aussi une grande erreur de croire que l'on puisse rançonner la classe paysanne, $[\ldots]$ ruiner les classes moyennes ${ }^{30}$."

Environ un an plus tard, Pierre-Étienne Flandin dit s'exprimer « au nom de 196 députés ". Il répond alors par un non-recevoir à l'ouverture, tentée par Léon Blum en mars 1938, du parti communiste aux modérés.

"Les groupes de la minorité, martèle l'interpellateur, précisent que la formule d'un rassemblement national autour du Front populaire qui leur a été seulement proposée et qui constituerait, en fait, un ralliement de l'opposition au programme de Front populaire, est inacceptable ${ }^{31}$."

Paul Reynaud est vertement tancé par ses pairs de l'Alliance pour avoir tenté de négocier avec Léon Blum.

De ces rappels, il s'ensuit que Pierre-Étienne Flandin se pose bien en leader de la minorité parlementaire hostile au Front populaire, en rassemblant des députés de l'opposition, et aussi en interprète du Français moyen. L'arrivée au pouvoir du Front populaire, la guerre d'Espagne - malgré la non-intervention prônée par Léon Blum - ne vont pas sans modifier la politique extérieure des alliancistes.

29. Ces quatre brochures sont conservées à la BDIC de Nanterre.

30. "L'interpellation de Flandin ", $A D, 5$ mars 1937.

31. "Au nom de 196 députés Pierre-Étienne Flandin définit l'attitude de la minorité (17 mars) ", $A D, 18,25$ mars 1938. 


\section{Pierre-Étienne Flandin leader du pacifisme de droite}

Après la première crise tchécoslovaque et au moment des accords de Munich, l'affrontement droite/gauche laisse place au clivage pacifistes/bellicistes. En réalité, au sein de la famille des droites et du centre droit, une divergence se produit entre partisans de la paix et opposants aux accords signés, en Allemagne, par Daladier.

En septembre 1938, Pierre-Étienne Flandin apparaît à la pointe du clan des pacifistes. N'adjure-t-il pas, par voie d'affiche, le gouvernement Daladier de signer l'accord? N'a-t-il pas, l'accord signé, envoyé un télégramme de félicitation aux quatre signataires, donc à Hitler? Un tel comportement induit non seulement la critique virulente d'Henri de Kérillis et du " camp de l'antifascisme international ", dixit Flandin, mais aussi une scission au sein de l'Alliance démocratique. Une minorité d'alliancistes, réprouvant l'action du président, a préféré quitter le parti, Paul Reynaud en tête; cependant, lors du congrès de Paris de novembre 1938, la majorité a voté une approbation à son leader.

Pierre-Étienne Flandin et les flandinistes ont été amenés à justifier cette politique que d'aucuns peuvent qualifier de défaitiste. Le président de l'Alliance fait état, dans une lettre adressée par voie de presse à Henri de Kérillis, leader des républicains nationaux, hostiles à Munich, que des informations alarmistes recueillies à Berlin, dès 1937, commandent sa politique de prudence. Au vrai, l'obsession moscoutaire oriente la conduite des flandinistes. Ceux-ci dénoncent "le bellicisme d'une gauche inféodée à Moscou ".

Dans cette conjecture " on n'a pas le droit de déclarer la guerre ", car " c'eût été faire le jeu d'une puissance étrangère ». Ils souscrivent au raisonnement du colonel Fabry, membre éminent de l'Alliance. Selon ce dernier :

" La Russie craint l'Allemagne et essaie de détourner l'orage. Et puis la Russie des Soviets, qui est sortie de la guerre de 1914-1918, peut penser que d'une nouvelle guerre européenne sortirait l'Europe des Soviets. "

Entre congressistes, les partisans de Pierre-Étienne Flandin n'hésitent également pas à évoquer l'impréparation de l'armée française et l'impuissance d'une nation déchirée par des luttes intestines :

"Le vrai patriotisme consiste, en effet, à ne pas lancer notre pays dans une aventure dont il pourrait sortir diminué."

Cependant, dans un ordre du jour paru dans l'AD du 18 octobre 1938, le comité directeur

"prend acte des accords de Munich, mais souligne que, s'ils ont aujourd'hui médiocrement assuré la paix, ils ne l'ont point garantie pour demain $[\ldots]^{32}$."

32. Remond, René et BouRdin, Janine, op. cit. et divers numéros de l'AD, en particulier $A D$ du 30 septembre et du 14 octobre. 
Quoi qu'il en soit de la crise interne de l'Alliance, Flandin apparaît lors de Munich, comme le porte-drapeau d'une droite antimoscoutaire et pacifiste. Les divisions internes permettent de mieux comprendre deux événements ultérieurs. Au moment de la déclaration de guerre, en 1939, l'Alliance démocratique éprouve quelques difficultés à se situer. Puis, en juillet 1940, la majorité des députés inscrits au groupe des Républicains de gauche et républicains radicaux, émanation de l'Alliance à la Chambre des députés, soit 34 sur 42, a voté les pleins pouvoirs à Pétain. "L'ambiguïté n'avait plus cours ", cette ambiguïté ou ambivalence caractéristique des alliancistes ${ }^{33}$.

Au banquet de clôture du congrès de novembre 1937, Léon Baréty constate :

" Il y a place sur l'échiquier politique pour un parti du centre. "In medio stat virtus", disait le poète latin. C'est en quelque sorte la devise de l'Alliance. En refusant les excès, elle ne s'enferme pas dans un conservatisme étroit ${ }^{34}$. "

En refusant de vraiment rallier les regroupements esquissés à droite, les instances dirigeantes de l'Alliance, à la fin des années trente ont, sans nul doute, failli à la remobilisation des droites, mais ont ainsi contribué à faire reculer l'échéance fascisante en France. Pourtant, au temps du Front populaire, elles ont été amenées à prendre parti dans le camp des droites contre celui des gauches, voire à entraîner le premier pas vers un pacifisme défaitiste, annonciateur de l'armistice de 1940.

Si Pierre-Étienne Flandin et nombre d'élus ont opté pour Pétain, ce choix, en fait, transcende quant à lui, le clivage droite/gauche même si, cela va de soi, la " révolution nationale " n'est pas équivoque.

33. Olivier WIeVORSKA, "Flandinistes dans la tourmente (1940-1944)", François Roth, Les Modérés dans la vie politique française (1870-1965), PUN, 2000.

34. «Discours de Louis Baréty au banquet de clôture du congrès ", AD, 26 novembre 1937. 


\section{RESUME}

P.-E. Flandin préside l'Alliance, née en 1901, à partir de 1933. Il situe son parti au centre de l'échiquier politique. C'est pourquoi il entraîne la majorité des alliancistes à repousser tout accord avec les autres forces de droite, et se présente, lui et son mouvement, comme les champions de l'opposition au Front populaire et du pacifisme de droite au moment des accords de Munich.

\section{ABSTRACT}

The Democratic Alliance and the rights recomposition

P.-E. Flandin chairs the Alliance, created in 1901, from 1933. He situates his party at the centre of the political chessboard. That's why he pulls the majority of the Alliancers to refuse any agreement with the other right forces, and stands, he and his movement, as the champions of the opposition to the Front populaire and of the right-wing pacifism at the moment of the Munich agreement. 